\title{
The efficacy and safety of simultaneous integrated dose reduction in clinical target volume with intensity-modulated radiotherapy for patients with locally advanced esophageal squamous cell carcinoma
}

\author{
Jingjing Zhao ${ }^{1 \#}$, Tongda Lei ${ }^{1 \#}$, Tian Zhang ${ }^{1}$, Xi Chen $^{1}$, Jie Dong ${ }^{2}$, Yong Guan ${ }^{1}$, Jing Wang ${ }^{1}$, Hui Wei ${ }^{1}$, \\ Puchun Er ${ }^{1}$, Dong Han ${ }^{1}$, Xiaoying Wei ${ }^{1}$, Zhoubo Guo ${ }^{1}$, Qingwu Du ${ }^{1}$, Jun Wang ${ }^{1}$, Ningbo Liu ${ }^{1}$, \\ Yongchun Song ${ }^{1}$, Zhiyong Yuan ${ }^{1}$, Lujun Zhao ${ }^{1}$, Wencheng Zhang ${ }^{1}$, Qingsong Pang ${ }^{1}$, Ping Wang ${ }^{1}$ \\ ${ }^{1}$ Department of Radiation Oncology, Tianjin Medical University Cancer Institute and Hospital, National Clinical Research Center for Cancer, Key \\ Laboratory of Cancer Prevention and Therapy, Tianjin, Tianjin's Clinical Research Center for Cancer, Tianjin, China; ${ }^{2}$ Department of Nutritional \\ Therapy, Tianjin Medical University Cancer Institute and Hospital, National Clinical Research Center for Cancer, Key Laboratory of Cancer \\ Prevention and Therapy, Tianjin, Tianjin's Clinical Research Center for Cancer, Tianjin, China \\ Contributions: (I) Conception and design: P Wang, Q Pang, W Zhang; (II) Administrative support: P Wang, Q Pang, W Zhang; (III) Provision \\ of study materials or patients: Q Pang, W Zhang, P Wang; (IV) Collection and assembly of data: J Zhao, W Zhang, T Lei; (V) Data analysis and \\ interpretation: J Zhao, T Lei; (VI) Manuscript writing: All authors; (VII) Final approval of manuscript: All authors. \\ \#These authors contributed equally to this work. \\ Correspondence to: Ping Wang; Qingsong Pang; Wencheng Zhang. Department of Radiation Oncology, Tianjin Medical University Cancer \\ Institute and Hospital/National Clinical Research Center for Cancer, Tianjin, China. Email: wangping@tjmuch.com; pangqingsong@tjmuch.com; \\ zhangwencheng@tjmuch.com.
}

Background: Compelling research to explore the effectiveness of simultaneous integrated dose reduction in clinical target volume (CTV) with intensity-modulated radiotherapy (SIR-IMRT) for locally advanced esophageal squamous cell carcinoma (ESCC) are limited. This study aimed to compare the clinical efficacy and treatment-related toxicity between SIR-IMRT and conventional IMRT (C-IMRT) in the treatment of ESCC.

Methods: From March 2010 to September 2016, the clinical data of 257 patients with ESCC who received definitive IMRT in the Tianjin Medical University Cancer Institute and Hospital were collected and retrospectively analyzed. Among these patients, 137 patients received C-IMRT with a prescribed dose of $60 \mathrm{~Gy}$ in 30 fractions for planning target volume (PTV), while 120 patients received SIR-IMRT with a prescribed dose of 60 Gy in 30 fractions for the planning gross tumor volume (PGTV) and a prescribed dose of $54 \mathrm{~Gy}$ in 30 fractions for PTV. All of the patients received definitive IMRT with elective nodal irradiation. Locoregional control, survival, treatment toxicity and dose to organs at risk (OAR) were compared between the groups.

Results: Patients who received SIR-IMRT showed a similar locoregional failure rate compared to the C-IMRT group (27.5\% versus $29.9 \%, \mathrm{P}=0.668)$. The 1 -, 2 - and 3 -year overall survival (OS) rates were $71.5 \%, 44.3 \%, 44.3 \%$ vs. $77.9 \%, 52.1 \%, 32.9 \%$ in the C-IMRT and SIR-IMRT groups, respectively $(\mathrm{P}=0.825)$. No significant differences were observed in PFS and LRRFS between the two groups $(\mathrm{P}=0.880$ and $\mathrm{P}=0.216$, respectively). The dose of lung V30 and the maximum dose of spinal cord in the C-IMRT group were significantly higher than those in the SIR-IMRT group ( $\mathrm{P}=0.013, \mathrm{P}=0.047)$. The incidence of acute radiation esophagitis was significantly lower in the SIR-IMRT group ( $\mathrm{P}=0.046)$, although no statistical difference was observed in the incidence of acute severe adverse events between the two groups.

Conclusions: SIR-IMRT offers an effective and safe option for patients with unresectable ESCC who receive definitive RT. Further prospective and larger sample size studies are warranted to confirm our results. 
Keywords: Esophageal squamous cell carcinoma (ESCC); intensity-modulated radiotherapy (IMRT); simultaneous integrated dose reduction; survival; toxicity

Submitted Apr 09, 2020. Accepted for publication Aug 14, 2020.

doi: $10.21037 / \mathrm{atm}-20-4366$

View this article at: http://dx.doi.org/10.21037/atm-20-4366

\section{Introduction}

Esophageal cancer (EC) is one of the most leading cause of cancer mortality worldwide (1). For patients at early clinical stage, surgical resection remains the main treatment. Several robust data have suggested that pre-operative chemoradiotherapy followed by surgery improved overall survival (OS) relative to surgery alone for patients with localized, operable EC $(2,3)$. While many EC patients are inoperable at the time of diagnosed or refuse surgery for personal reasons. For these patients, concurrent chemoradiotherapy (CCRT) is the standard care (4).

In Western countries, the predominant histological type of $\mathrm{EC}$ is adenocarcinoma, whereas in China, squamous cell carcinoma (SCC) is the most common type of EC $(5,6)$. These two types exhibit different biological characteristics and treatment response $(7,8)$. EC patients who undergo definitive CCRT in Europe and North America typically receive a total dose of $50.4 \mathrm{~Gy}$, which has been accepted as the standard radiation dose based on the results of the Radiation Therapy Oncology Group (RTOG)-8501 (4) and RTOG 94-05 (9). Meanwhile, in China, a dose of $60 \mathrm{~Gy}$ is preferred, owing to the high local recurrence rate after receiving $\mathrm{RT}$ with the recommended standard radiation dose (10). However, some studies have suggested that the increased radiation-induced toxicity, which can lead to decreased treatment intensification, should not be ignored $(11,12)$. The optimal radiation dose for esophageal squamous cell carcinoma (ESCC) has been a topic of clinical investigation in an attempt to achieve better survival outcomes for many years.

Conventional intensity-modulated radiotherapy (C-IMRT) delivers same radiation dose to gross tumor volume (GTV) and clinical target volume (CTV). With the remarkable development of RT technology, modern radiation techniques offer the advantage of optimizing radiation dose distribution by simultaneously conferring a higher RT dose to the gross tumor and a relatively lower dose to the subclinical disease (13-15). Advanced RT techniques seem to provide appropriate solution for the arguments of the optimal radiation dose for ESCC. In recent years, simultaneous integrated dose reduction in CTV with IMRT (SIR-IMRT) has been applied widely in clinical settings for the curative treatment for ESCC, with the treatment planning of selectively delivering definitive radiation dose of 60 Gy to the GTV and a lower dose of 54 Gy to the CTV, which potentially reduces irradiation toxicity. Although previous studies have reported that 45-50 Gy could effectively eradicate subclinical disease, no convincing data has compared the clinical outcomes between SIR-IMRT and C-IMRT for ESCC (16-18).

In this study, we retrospectively compared the clinical efficacy and treatment-related toxicity between SIR-IMRT and C-IMRT in the treatment of patients with inoperable ESCC.

We present the following article in accordance with the STROBE reporting checklist (available at http://dx.doi. org/10.21037/atm-20-4366).

\section{Methods}

\section{Data source}

The data in this study was obtained from Tianjin Medical University Cancer Hospital and included sufficient information on patient demographics, disease characteristics, treatment details, tumor control, survival outcomes, and toxicities. All procedures performed in this study involving human participants were in accordance with the Declaration of Helsinki (as revised in 2013). The study was approved by the Ethics Committee of Tianjin Medical University Cancer Institute and Hospital (No. bc2020052). Patient data was retrieved from hospital medical record system, so an informed consent form was not required. The patient's personal data has been secured.

\section{Patient selection and study design}

The study flow diagram is shown in Figure 1. A total of 579 EC patients who received radical RT at the hospital between March 2010 and September 2016 were enrolled as the initial study population. The eligibility criteria were 
as follows: (I) aged $\geq 18$ years; (II) pathologically confirmed as ESCC; (III) clinical stage of TanyNanyM $M_{0}$ or $M_{1}$ only with supraclavicular or abdominal lymph node metastasis, based on the 6th edition of the American Joint Committee on Cancer (AJCC 6th) tumor node metastasis (TNM) stage classification; (IV) with an inoperable tumor or refused surgery; (V) underwent radical IMRT or IMRT-based chemoradiotherapy; (VI) Karnofsky performance status (KPS) score $\geq 70$; and (VII) complete clinical data.

A total of 518 EC patients treated with definitive IMRT met the above criteria, and they were excluded if they met the following criteria: (I) non-SCC histology or other coexisting primary tumors; (II) prior thoracic radiotherapy or surgery; (III) radiation dose (delivered to PTV) <60 Gy

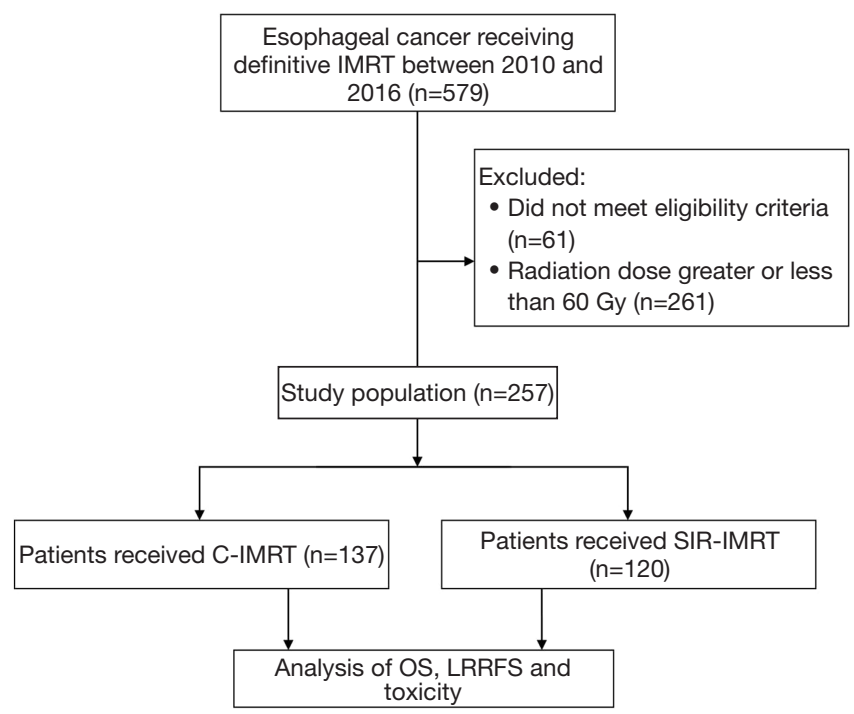

Figure 1 Study flow diagram. SIR-IMRT, simultaneous integrated dose reduction in clinical target volume with intensity-modulated radiotherapy; C-IMRT, conventional-IMRT. or >60 Gy; (V) unconventional dose fractional RT.

Finally, a total of 257 patients were selected and categorized into 2 groups based on radiation modality: 137 patients in the C-IMRT group, and 120 patients in the SIR-IMRT group. For patients who received conventional IMRT, the prescribed radiation dose was 60 Gy to the planning target volume (PTV). For patients with SIRIMRT, the GTV received the same prescribed dose as the C-IMRT group, while the doses to the CTV and PTV were simultaneously decreased to 54 Gy. Table 1 summarizes the general clinical data of both groups.

\section{Treatment strategy}

All enrolled patients underwent radical IMRT with elective node irradiation. The GTV included any visible primary tumor as well as any involved regional lymph nodes detected by CT, esophagogram, and endoscopy. To create the CTV, the GTV was expanded by a margin of 3.0 and $0.6 \mathrm{~cm}$ at the long and lateral axes, respectively. A margin of $0.5 \mathrm{~cm}$ around the CTV was drawn to outline the PTV. The planning GTV (PGTV) was reached uniformly by expanding by $0.5 \mathrm{~cm}$ around the GTV in the SIR-IMRT group only.

In the C-IMRT group, the prescribed dose was $60 \mathrm{~Gy}$ in 30 fractions of 2.0 Gy per fraction. In the SIR-IMRT group, the prescribed dose was $60 \mathrm{~Gy}$ in 30 fractions of 2.0 Gy per fraction to the GTV and PGTV, with a dose of 54 Gy in 30 fractions of 1.8 Gy per fraction to the CTV and PTV. All doses delivered met the requirement that $95 \%$ of the PGTV and PTV receive the prescribed dose. The dose constraints to organs at risk (OAR) were as follows: lung V20 $\leq 30 \%, \mathrm{~V} 30 \leq 20 \%$, and the mean lung dose $\leq 16$ Gy; heart $\mathrm{V} 30 \leq 40 \%, \mathrm{~V} 40 \leq 30 \%$, and the mean heart dose $\leq 28 \mathrm{~Gy}$; and the maximum dose to the spinal cord $<45$ Gy. A total of 215 patients received concurrent or

Table 1 Baseline patient and disease characteristics

\begin{tabular}{|c|c|c|c|c|}
\hline Characteristics & Total, 257 (100.0\%) & C-IMRT group [n=137 (\%)] & SIR-IMR group [n=120 (\%)] & $P$ value \\
\hline Gender & & & & 0.760 \\
\hline Male & $210(81.7)$ & $111(81.0)$ & $99(82.5)$ & \\
\hline Female & $47(18.3)$ & $26(19.0)$ & $21(17.5)$ & \\
\hline Age, years & & & & 0.110 \\
\hline$<65$ & $158(61.5)$ & 78 (56.9) & $80(66.7)$ & \\
\hline$\geq 65$ & $99(38.5)$ & $59(43.1)$ & $40(33.3)$ & \\
\hline
\end{tabular}

Table 1 (continued) 
Table 1 (continued)

\begin{tabular}{|c|c|c|c|c|}
\hline Characteristics & Total, 257 (100.0\%) & C-IMRT group [n=137 (\%)] & SIR-IMR group [n=120 (\%)] & $P$ value \\
\hline$\geq 90$ & $163(63.4)$ & $86(62.8)$ & $77(64.2)$ & \\
\hline$<90$ & $94(36.6)$ & $51(37.2)$ & $43(35.8)$ & \\
\hline Smoking & & & & 0.362 \\
\hline Yes & $166(64.6)$ & $85(62.0)$ & $81(67.5)$ & \\
\hline Tumor location & & & & 0.426 \\
\hline Cervical & $21(8.2)$ & $12(8.8)$ & $9(7.5)$ & \\
\hline Upper thoracic & $91(35.4)$ & $54(39.4)$ & $37(30.8)$ & \\
\hline AJCC clinical stage & & & & 0.601 \\
\hline II & $50(19.5)$ & $26(19.0)$ & $24(20.0)$ & \\
\hline III & $131(51.0)$ & $75(54.7)$ & $56(46.7)$ & \\
\hline IV & $76(29.6)$ & $36(26.3)$ & 40 (33.3) & \\
\hline T stage & & & & 0.268 \\
\hline $\mathrm{T} 2$ & $24(9.3)$ & $11(8.0)$ & $13(10.8)$ & \\
\hline T3 & $76(29.6)$ & $36(26.3)$ & $40(33.3)$ & \\
\hline $\mathrm{T} 4$ & $157(61.1)$ & $90(65.7)$ & $67(55.8)$ & \\
\hline$>5$ & $140(54.5)$ & $79(57.7)$ & $61(50.8)$ & \\
\hline GTV volume, $\mathrm{cm}^{3}$ & & & & 0.427 \\
\hline$\leq 40$ & $141(54.9)$ & $72(52.6)$ & 69 (57.5) & \\
\hline$>40$ & $116(45.1)$ & $65(47.4)$ & $51(42.5)$ & \\
\hline Chemotherapy & & & & 0.586 \\
\hline No & $42(16.3)$ & $24(17.5)$ & $18(15.0)$ & \\
\hline Yes & 215 (83.7) & $113(82.5)$ & $102(85.0)$ & \\
\hline CCRT & & & & 0.582 \\
\hline No & $88(34.2)$ & 49 (35.8) & $39(32.5)$ & \\
\hline Yes & $169(65.8)$ & $88(64.2)$ & $81(67.5)$ & \\
\hline
\end{tabular}

SIR-IMRT, simultaneous integrated dose reduction in clinical target volume with intensity-modulated radiotherapy; C-IMRT, conventionalIMRT; KPS, Karnofsky performance status; AJCC, American Joint Committee Cancer; GTV, gross tumor volume; CCRT, concurrent chemoradiotherapy. 
Table 2 Comparison of first failure patterns of patients between the C-IMRT and SIR-IMRT groups

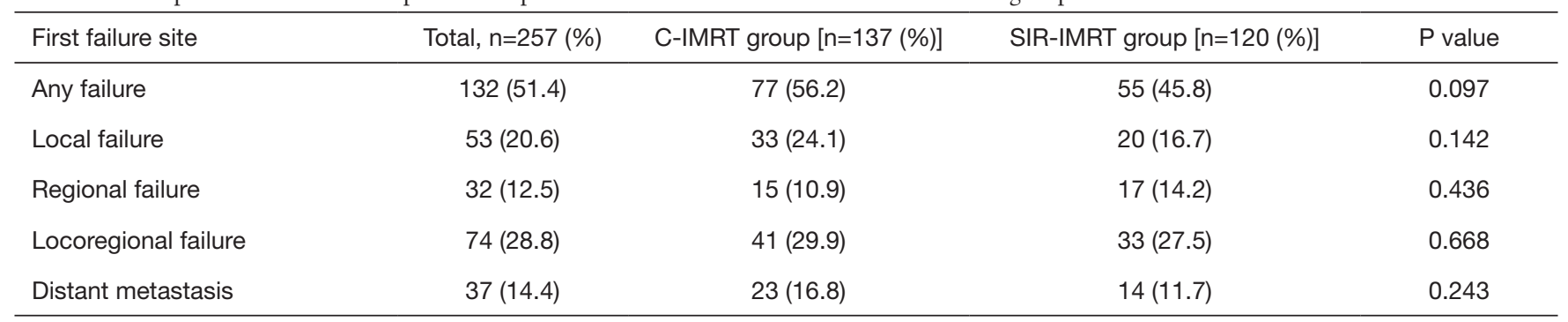

SIR-IMRT, simultaneous integrated dose reduction in clinical target volume with intensity-modulated radiotherapy; C-IMRT, conventionalIMRT.

sequential platinum-based chemotherapy, including 5-FUcisplatin, paclitaxel-cisplatin, oxaliplatin-capecitabine.

\section{End points}

Local/regional failure was defined as the persistence or recurrence of the primary tumor or regional lymph nodes. The end points included OS, progression-free survival (PFS), locoregional recurrence-free survival (LRRFS), and treatment-related toxicities. OS was defined as the length of time from the date of first treatment to the date of the last follow-up or death from any cause. PFS was defined from the time of first treatment to disease progression, and LRRFS was defined as the time to recurrences of primary tumor or regional lymph nodes. Patients who did not experience disease progression were recorded at the date of death or last follow-up. Treatment-related toxicities were evaluated according to the Common Terminology Criteria for Adverse Events (CTCAE) 4.0 criteria.

\section{Statistical analysis}

The Kaplan-Meier method was used to assess OS, PFS, and LRRFS and curves were compared by log-rank tests. Dichotomous data between groups were compared by chisquare test. Continuous variables were compared by using the Mann-Whitney $U$ test. All statistical analyses were carried out using SPSS software (version 24.0, IBM SPSS, CA, USA). A two-sided $\mathrm{P}$ value of $<0.05$ was considered to represent statistical significance.

\section{Results}

\section{Patient characteristics}

A total of 257 ESCC patients who underwent IMRT were enrolled in this study including 137 with C-IMRT and 120 with SIR-IMRT. The characteristics of the 257 eligible patients are summarized in Table 1. No significant differences were found between the two groups in relation to the distribution of age, gender, KPS score, cigarette smoking, tumor location, clinical stage, tumor length, GTV volume, or treatment with chemotherapy/ CCRT or not $(\mathrm{P}>0.05$, Table 1).

\section{Patterns of first failure}

During the follow-up period, 132 (51.4\%) of the 257 patients experienced disease progression: 53 (20.6\%) developed first failure at the local site alone, 32 (12.5\%) experienced at the regional site, 74 (28.8\%) had local, regional, or local-regional (locoregional) recurrence, and 37 (14.4\%) experienced distant failure. There were no significant differences in the incidence of local $(\mathrm{P}=0.142)$, regional $(\mathrm{P}=0.436)$, locoregional $(\mathrm{P}=0.668)$, or distant $(\mathrm{P}=0.243)$ first progression between the C-IMRT and SIR-IMRT group. The distribution of first progression is summarized in Table 2.

\section{Local control and survival}

At a median follow-up of 19.6 months, the 1-, 2-, and 3 -year OS rates for the entire cohort were $74.1 \%, 47.0 \%$, and $41.1 \%$, respectively. The 1-, 2-, and 3-year PFS rates were $57.2 \%, 36.7 \%$, and $33.3 \%$, respectively; and the 1-, 2-, and 3-year LRRFS rates were $80.8 \%, 59.0 \%$, and $50.7 \%$, respectively. The median OS, PFS, and LRRFS were 20.5, 14.3, and 39.0 months, respectively. In the C-IMRT group and the SIR-IMRT group, the 1-, 2-, 3-year OS rates were $71.5 \%, 44.3 \%$, vs. $44.3 \%$, and $77.9 \%, 52.1 \%$, and $32.9 \%$, respectively $(\mathrm{P}=0.825)$ (Figure $2 A)$, and the $1-, 2-$, and 3 -year PFS rates were $57.1 \%, 34.1 \%, 34.1 \%$ vs. $57.3 \%$, 

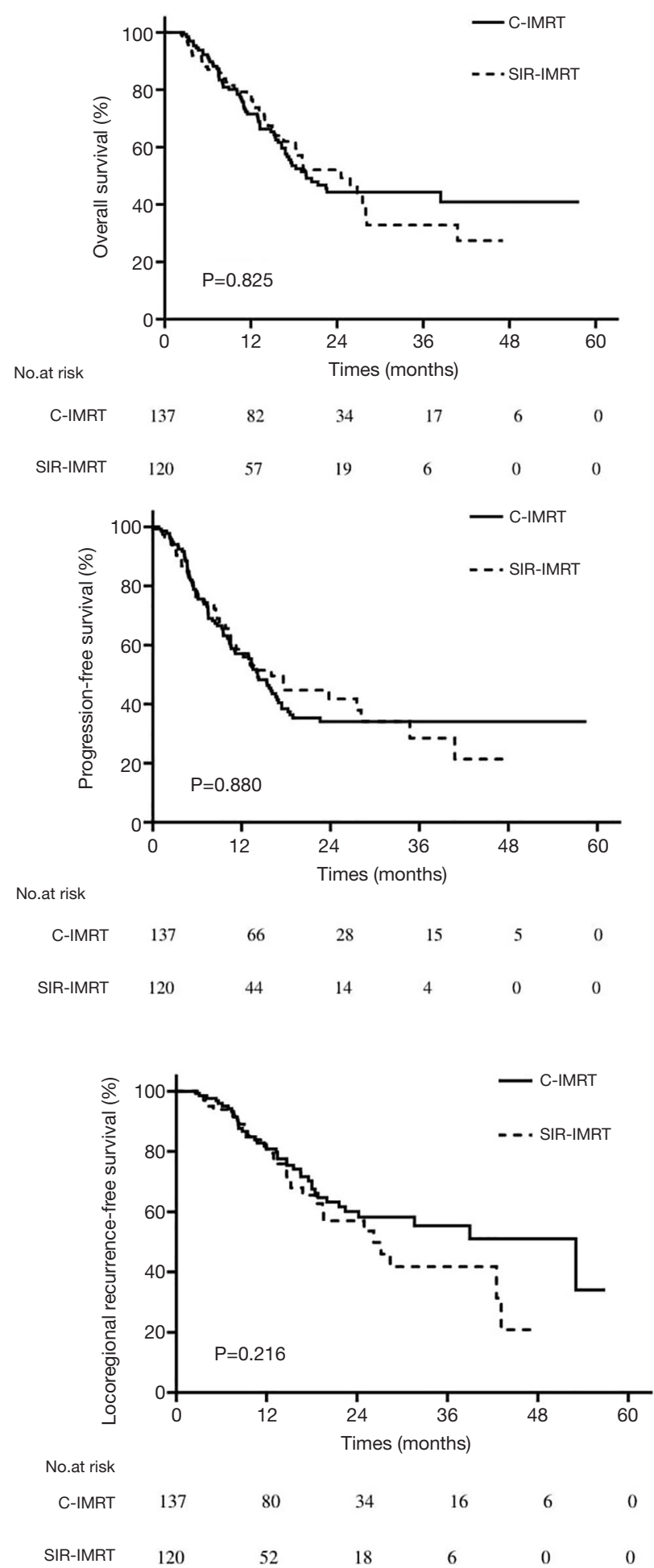

Figure 2 Kaplan-Meier curves for (A) overall survival, (B) progression-free survival, and $(\mathrm{C})$ locoregional recurrence-free survival. SIR-IMRT, simultaneous integrated dose reduction in clinical target volume with intensity-modulated radiotherapy; C-IMRT, conventional-IMRT.
$41.8 \%, 28.5 \%$, respectively $(\mathrm{P}=0.880)$ (Figure $2 B)$. The 1 -, $2-$, and 3-year LRRFS rates were $80.8 \%, 60.0 \%, 55.4 \%$, respectively, in the C-IMRT group, and $80.8 \%, 57.0 \%$, and $41.8 \%$, respectively, in the SIR-IMRT group $(\mathrm{P}=0.216)$ (Figure 2C). No significant differences were observed in OS, PFS, or LRRFS between the two groups (Figure 2).

\section{Subgroup analyses of locoregional control and OS}

Table 3 lists the results of stratified analysis of locoregional control and OS in the C-IMRT and SIR-IMRT groups. For ESCC patients with GTV volume $>40 \mathrm{~cm}^{3}$, no difference was observed in the rate of locoregional control between the two groups $(\mathrm{P}=0.317)$, while the $\mathrm{C}-\mathrm{IMRT}$ group had a slightly better rate of OS than the SIR-IMRT group $(\mathrm{P}=0.069)$. C-IMRT was also associated with relatively higher rates of locoregional control and OS in patients who did not undergo chemotherapy $(\mathrm{P}=0.087$ and $\mathrm{P}=0.056$, respectively) or concurrent chemotherapy $(\mathrm{P}=0.066$ and $\mathrm{P}=0.051$, respectively), although the differences were not statistically significant.

\section{Toxicities and dose of OARs}

The incidence and severity of treatment-related toxicities are shown in Table 4. The incidence of acute radiation esophagitis in patients who received SIR-IMRT was significantly lower than in those who received C-IMRT ( $\geq$ grade $2,35.0 \%$ vs. $46.7 \%, \mathrm{P}=0.046$ ). Thrombocytopenia was more common in the C-IMRT group than in the SIR-IMRT group, but the difference was not statistically significant $(\mathrm{P}=0.068)$. No significant differences were observed between the rates of other toxicities of any grade in the two groups, including radiation pneumonitis and hematologic toxicities.

Acute severe adverse events occurred at a low frequency in both groups and are detailed in Table 5. In patients who received C-IMRT or SIR-IMRT, statistically similar rates of radiation esophagitis $(8.0 \%$ vs. $4.2 \%, \mathrm{P}=0.622)$, radiation pneumonitis $(0.7 \%$ vs. $0 \%)$, and myelosuppression $(12.4 \%$ vs. $7.5 \%, \mathrm{P}=0.169)$ of grade 3 and above were observed.

Compared with the delivered dose to OARs in the C-IMRT group, the lung V30 and the maximum dose to the spinal cord in the SIR-IMRT group were significantly reduced $(\mathrm{P}=0.013$ and $\mathrm{P}=0.047$, respectively). No differences were observed in lung doses (V5, V20, mean dose) or heart doses (V30, V40, mean dose) between the two groups. The dose parameters of OARs in both groups are presented in Table 6 . 
Table 3 Subgroup analyses of locoregional control and overall survival

\begin{tabular}{|c|c|c|c|c|c|c|c|c|}
\hline Characteristics & \multicolumn{3}{|c|}{ Locoregional control (\%) } & $\mathrm{P}$ value & \multicolumn{3}{|c|}{ Overall survival (\%) } & $P$ value \\
\hline Cervical/upper esophagus & & & & & & & & 0.421 \\
\hline C-IMRT & 85.2 & 56.3 & 56.3 & 0.160 & 82.5 & 53.2 & 53.2 & \\
\hline SIR-IMRT & 75.9 & 52.9 & 40.8 & & 85.4 & 56.7 & 28.8 & \\
\hline C-IMRT & 75.2 & 46.5 & 46.5 & 0.710 & 62.8 & 35.7 & 35.7 & \\
\hline SIR-IMRT & 82.4 & 57.0 & 42.3 & & 74.4 & 48.4 & 31.4 & \\
\hline Early stage (II) & & & & & & & & 0.941 \\
\hline C-IMRT & 86.7 & 74.8 & 49.9 & 0.768 & 76.0 & 61.9 & 61.9 & \\
\hline C-IMRT & 79.3 & 56.4 & 56.4 & 0.213 & 70.2 & 40.0 & 40.0 & \\
\hline SIR-IMRT & 75.6 & 57.0 & 42.1 & & 72.7 & 53.9 & 32.0 & \\
\hline AJCC T2 stage & & & & & & & & 0.189 \\
\hline C-IMRT & 87.5 & 70.0 & 70.0 & 0.991 & 70.0 & 56.0 & 56.0 & \\
\hline SIR-IMRT & 100.0 & 83.3 & 66.7 & & 100.0 & 83.3 & 66.7 & \\
\hline AJCC T3-T4 stage & & & & & & & & 0.386 \\
\hline C-IMRT & 80.2 & 59.4 & 54.5 & 0.191 & 71.6 & 43.4 & 43.4 & \\
\hline SIR-IMRT & 77.8 & 52.6 & 37.5 & & 74.9 & 47.5 & 26.5 & \\
\hline SIR-IMRT & 75.9 & 52.9 & 40.8 & & 72.7 & 51.0 & 29.2 & \\
\hline Tumor length $\leq 5 \mathrm{~cm}$ & & & & & & & & 0.421 \\
\hline C-IMRT & 85.2 & 56.3 & 56.3 & 0.160 & 82.5 & 53.2 & 53.2 & \\
\hline SIR-IMRT & 75.9 & 52.9 & 40.8 & & 85.4 & 56.7 & 28.8 & \\
\hline Tumor length $>5 \mathrm{~cm}$ & & & & & & & & 0.922 \\
\hline C-IMRT & 73.5 & 48.4 & 45.9 & 0.981 & 63.4 & 38.1 & 38.1 & \\
\hline SIR-IMRT & 73.6 & 49.1 & 49.1 & & 70.6 & 46.0 & 34.5 & \\
\hline GTV volume $\leq 40 \mathrm{~cm}^{3}$ & & & & & & & & 0.275 \\
\hline C-IMRT & 79.4 & 62.9 & 52.6 & 0.431 & 71.4 & 43.8 & 43.8 & \\
\hline SIR-IMRT & 88.4 & 57.3 & 37.0 & & 89.8 & 58.7 & 38.1 & \\
\hline
\end{tabular}

Table 3 (continued) 
Table 3 (continued)

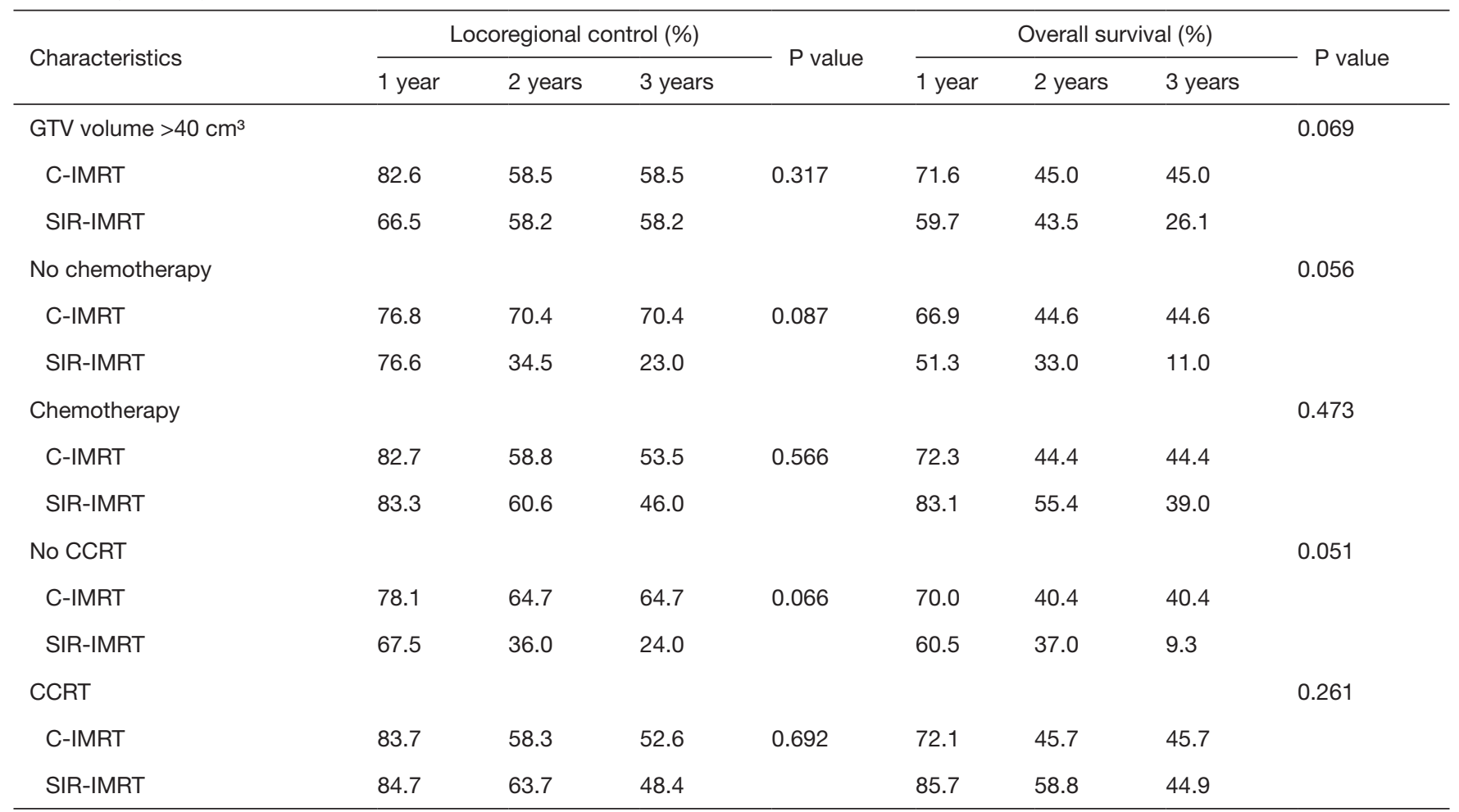

SIR-IMRT, simultaneous integrated dose reduction in clinical target volume with intensity-modulated radiotherapy; C-IMRT, conventionalIMRT; AJCC, American Joint Committee Cancer; GTV, gross tumor volume; CCRT, concurrent chemoradiotherapy.

Table 4 Comparison of acute toxicities between the C-IMRT and SIR-IMRT groups

\begin{tabular}{|c|c|c|c|c|c|c|c|c|c|}
\hline Toxicities & \multicolumn{4}{|c|}{ C-IMRT group [n=137 (\%)] } & \multicolumn{4}{|c|}{ SIR-IMRT [n=120 (\%)] } & $P$ value \\
\hline Radiation esophagitis & $73(53.3)$ & $53(38.7)$ & $8(5.8)$ & $3(2.2)$ & $78(65.0)$ & $37(30.8)$ & $3(2.5)$ & $2(1.7)$ & 0.046 \\
\hline Radiation pneumonitis & $133(97.1)$ & $3(2.2)$ & $1(0.7)$ & $0(0.0)$ & $111(92.5)$ & $9(7.5)$ & $0(0.0)$ & $0(0.0)$ & 0.099 \\
\hline Leukopenia & 99 (72.3) & $26(19.0)$ & $12(8.8)$ & $0(0.0)$ & $87(72.5)$ & $29(24.2)$ & $4(3.3)$ & $0(0.0)$ & 0.761 \\
\hline Anemia & $119(86.9)$ & 15 (10.9) & $3(2.2)$ & $0(0.0)$ & $109(90.8)$ & $9(7.5)$ & $2(1.7)$ & $0(0.0)$ & 0.319 \\
\hline Thrombocytopenia & $132(96.4)$ & $3(2.2)$ & $2(1.5)$ & $0(0.0)$ & $109(90.8)$ & $7(5.8)$ & $4(3.3)$ & $0(0.0)$ & 0.068 \\
\hline Myelosuppression & $90(65.7)$ & $30(21.9)$ & $17(12.4)$ & $0(0.0)$ & 77 (64.2) & $34(28.3)$ & $8(6.7)$ & $1(0.8)$ & 0.968 \\
\hline
\end{tabular}

SIR-IMRT, simultaneous integrated dose reduction in clinical target volume with intensity-modulated radiotherapy; C-IMRT, conventionalIMRT.

\section{Discussion}

ESCC was reported to have high local failure rate and poor survival outcome with standard radiation dose of CCRT (7). Some studies have suggested that radiation dose escalation was an effective measure to improve local control, but the dose-reduced toxicity should not be underestimated $(8,9)$. Therefore, emerging researches focus on optimizing radiation delivery based on modern $\mathrm{RT}$ techniques $(10-12)$. In this study, we retrospectively analyzed the 
Table 5 Comparison of acute sever toxicities between the C-IMRT and SIR-IMRT groups

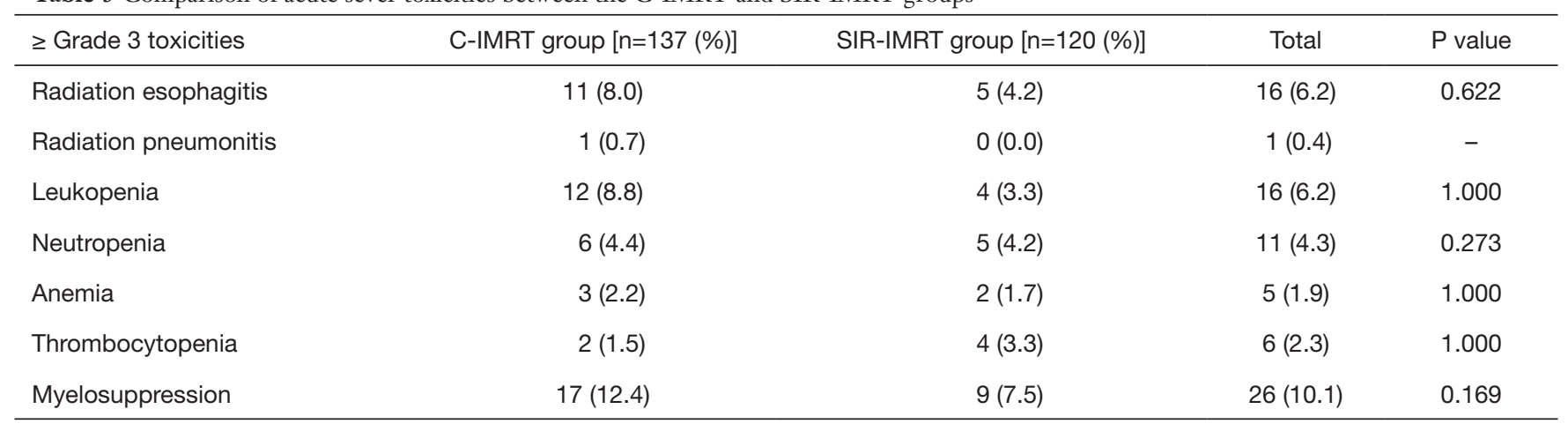

SIR-IMRT, simultaneous integrated dose reduction in clinical target volume with intensity-modulated radiotherapy; C-IMRT, conventionalIMRT.

Table 6 Comparison of radiation dose to OARs between the C-IMRT and SIR-IMRT groups

\begin{tabular}{lccc}
\hline Variables & C-IMRT group SIR-IMRT group & \\
& $(\mathrm{n}=137)$ & $(\mathrm{n}=120)$ & \\
\hline Mean lung dose (Gy) & $11.17 \pm 3.08$ & $11.30 \pm 5.51$ & 0.269 \\
Lung V5 (\%) & $43.72 \pm 12.41$ & $46.63 \pm 10.97$ & 0.074 \\
Lung V20 (\%) & $21.94 \pm 6.69$ & $21.61 \pm 6.43$ & 0.342 \\
Lung V30 (\%) & $13.17 \pm 4.94$ & $11.99 \pm 4.61$ & 0.013 \\
Mean heart dose (Gy) & $18.55 \pm 24.42$ & $19.80 \pm 12.48$ & 0.122 \\
Heart V30 (\%) & $23.89 \pm 19.57$ & $26.32 \pm 18.00$ & 0.290 \\
Heart V40 (\%) & $14.44 \pm 12.48$ & $15.56 \pm 12.26$ & 0.444 \\
Maximum spinal cord & $43.26 \pm 5.74$ & $43.11 \pm 4.75$ & 0.047 \\
dose (Gy) & & & \\
\hline
\end{tabular}

SIR-IMRT, simultaneous integrated dose reduction in clinical target volume with intensity-modulated radiotherapy; C-IMRT, conventional-IMRT; V5 Volumes receiving more than 5 Gy; V20 Volumes receiving more than $20 \mathrm{~Gy}$; V30 Volumes receiving more than 30 Gy V40 Volumes receiving more than 40 Gy.

clinical efficacy and safety of SIR-IMRT in the treatment of ESCC. The results demonstrated that SIR-IMRT provided similar locoregional control and survival outcomes compared to C-IMRT, with reduced toxicity for ESCC patients who received definitive RT. Our findings might put SIR-IMRT forward as an efficient and safe treatment option for patients with ESCC, although further confirmation in randomized controlled trials is merited.

CCRT has been widely accepted as the standard of care for patients with locally advanced EC. However, since the tumor local control and survival outcomes associated with CCRT are still poor, the optimal radical RT dose has long been the subject of intense debate (19-24). In China, where the predominant histological type of EC is SCC, a total radiation dose of $60 \mathrm{~Gy}$ is preferred for EC patients who undergo definitive treatment. SCC possesses different biological characteristics to adenocarcinoma, which accounts for the majority of EC cases in Western countries. Welsh et al. reported that $50 \%$ of EC patients experienced local failure after receiving definitive chemoradiotherapy with a total dose of 50.4 Gy. Of all cases of local failure, $90 \%$ were in the GTV, $23 \%$ in the CTV, and $12 \%$ in the PTV (10). This result demonstrated that the recommended dose of 50.4 Gy might be sufficient to achieve good control in subclinical areas, while local control can be improved by increasing the primary tumor radiation dose. Earlier studies also suggested that 45-50 Gy could effectively sterilize subclinical metastases, and a minimum of $60 \mathrm{~Gy}$ was required to control unresectable solid tumors (16-18). Hence, in the present study, SIR-IMRT simultaneously delivered radiation doses of 60 Gy to the primary tumor and $54 \mathrm{~Gy}$ to the subclinical regions.

In recent years, the rapid development of RT technology has seen three-dimensional treatment planning become widely applied in clinical settings. Such developments include IMRT, simultaneous integrated boost-IMRT (SIBIMRT), and volumetric-modulated arc therapy (VMAT), which can deliver a higher RT dose to the gross tumor and a lower dose to the surrounding normal tissue in a more precise manner than can be achieved by conventional RT (13-15). In this study, the doses to the CTV and PTV were significantly decreased in the SIR-IMRT group ( 54 vs. $60 \mathrm{~Gy}$ ), but there was no obvious increase in the rate of locoregional failure $(27.5 \%$ vs. $29.9 \%, \mathrm{P}=0.668)$, which indicated that 54 Gy was able to effectively control 
subclinical disease. In relation to treatment-related toxicity and the dose to OARs, patients with SIR-IMRT developed acute radiation esophagitis less frequently $(\mathrm{P}=0.046)$, and had significantly lower lung V30 (12.0\% vs. $13.2 \%$, $\mathrm{P}=0.013$ ) and maximum spinal cord volume (43.1 vs. 43.3 Gy, P=0.046) than the C-IMRT group. Severe adverse events occurred at a low frequency in both groups, and no significant difference was observed between two groups in rate of $\geq$ grade 3 toxicities. Furthermore, there was no statistical difference in long-term survival between SIRIMRT and C-IMRT group (2-year OS rate, $52.1 \%$ vs. $44.3 \%, \mathrm{P}=0.825)$. We can conclude based on these results that SIR-IMRT is a safe and effective option for patients with unresectable ESCC.

Data from Anderson Cancer Center in the United States showed that an SIB-IMRT plan of 64.8 Gy (28 fractions at 2.3 Gy per fraction) to the GTV and a standard dose of 50.4 Gy (28 fractions at 1.8 Gy per fraction) to the CTV and PTV could enable an escalated radiation dose to be safely delivered to the primary tumor, while simultaneously reducing the dose to proximal critical structures including the heart, lungs, and liver (15). A phase II trial conducted by $\mathrm{Yu}$ et al. suggested that patients treated with IMRT with prescribed doses of 50.4 Gy to the CTV (1.8 Gy/fraction) and 63 Gy to the GTV (2.25 Gy/fraction) achieved favorable locoregional control (3-year LRRFS, 67.5\%) with a low toxicity profile (25). A phase I/II trial that enrolled 46 patients with locally advanced EC who were treated with SIB-IMRT (63 Gy to the GTV and 50.4 Gy to the PTV) between 2010 and 2015, showed that the treatment protocols were well tolerated and achieved promising local control rates (with 6-month, 1-year, and 2-year local recurrence rates of $22 \%, 30 \%$, and $33 \%$, respectively) $(26,27)$. Our results were compatible with these studies. However, it is worth mentioning that in our study, patients who had large tumor burden $\left(\mathrm{GTV}>40 \mathrm{~cm}^{3}\right)$ or who did not receive chemotherapy/concurrent chemotherapy were more likely to benefit from C-IMRT. Therefore, individualized RT plans should be implemented in accordance with the specific conditions of patients in clinic.

This study was limited by its retrospective nature, which resulted in a lack of uniformity in the chemotherapy regimens of patients. Moreover, the relatively small number of patients enrolled might limit the generalizability of our conclusions. Our results must therefore be confirmed by prospective studies with larger sample sizes.

In summary, SIR-IMRT proved to be an effective and safe option for patients with unresectable EC treated by definitive RT. Its use should be further explored in largescale randomized controlled trials.

\section{Acknowledgments}

Funding: The study was supported the Clinical Trial Supporting Foundation of Tianjin Medical University Cancer Institute \& Hospital (Grant No. C1707).

\section{Footnote}

Reporting Checklist: The authors have completed the STROBE reporting checklist. Available at http://dx.doi. org/10.21037/atm-20-4366

Data Sharing Statement: Available at http://dx.doi. org/10.21037/atm-20-4366

Conflicts of Interest: All authors have completed the ICMJE uniform disclosure form (available at http://dx.doi. org/10.21037/atm-20-4366). The authors have no conflicts of interest to declare.

Ethical Statement: The authors are accountable for all aspects of the work in ensuring that questions related to the accuracy or integrity of any part of the work are appropriately investigated and resolved. All procedures performed in this study involving human participants were in accordance with the Declaration of Helsinki (as revised in 2013). The study was approved by the Ethics Committee of Tianjin Medical University Cancer Institute and Hospital (No. bc2020052). Patient data was retrieved from hospital medical record system, so an informed consent form was not required. The patient's personal data has been secured.

Open Access Statement: This is an Open Access article distributed in accordance with the Creative Commons Attribution-NonCommercial-NoDerivs 4.0 International License (CC BY-NC-ND 4.0), which permits the noncommercial replication and distribution of the article with the strict proviso that no changes or edits are made and the original work is properly cited (including links to both the formal publication through the relevant DOI and the license). See: https://creativecommons.org/licenses/by-nc-nd/4.0/.

\section{References}

1. Bray F, Ferlay J, Soerjomataram I, et al. Global cancer 
statistics 2018: GLOBOCAN estimates of incidence and mortality worldwide for 36 cancers in 185 countries. CA Cancer J Clin 2018;68:394-424.

2. Shapiro J, van Lanschot JJB, Hulshof M, et al. Neoadjuvant chemoradiotherapy plus surgery versus surgery alone for oesophageal or junctional cancer (CROSS): long-term results of a randomised controlled trial. Lancet Oncol 2015;16:1090-8.

3. Yang H, Liu H, Chen Y, et al. Neoadjuvant Chemoradiotherapy Followed by Surgery Versus Surgery Alone for Locally Advanced Squamous Cell Carcinoma of the Esophagus (NEOCRTEC5010): A Phase III Multicenter, Randomized, Open-Label Clinical Trial. J Clin Oncol 2018;36:2796-803.

4. Cooper JS, Guo MD, Herskovic A, et al. Chemoradiotherapy of locally advanced esophageal cancer: long-term follow-up of a prospective randomized trial (RTOG 85-01). Radiation Therapy Oncology Group. JAMA 1999;281:1623-7.

5. Ajani JA. Gastroesophageal cancers: progress and problems. J Natl Compr Canc Netw 2008;6:813-4.

6. Li H, Fang W, Yu Z, et al. Chinese expert consensus on mediastinal lymph node dissection in esophagectomy for esophageal cancer (2017 edition). J Thorac Dis 2018;10:2481-9.

7. Song Y, Li L, Ou Y, et al. Identification of genomic alterations in oesophageal squamous cell cancer. Nature 2014;509:91-5.

8. Domper Arnal MJ, Ferrández Arenas Á, Lanas Arbeloa Á. Esophageal cancer: Risk factors, screening and endoscopic treatment in Western and Eastern countries. World J Gastroenterol 2015;21:7933-43.

9. Minsky BD, Pajak TF, Ginsberg RJ, et al. INT 0123 (Radiation Therapy Oncology Group 94-05) phase III trial of combined-modality therapy for esophageal cancer: high-dose versus standard-dose radiation therapy. J Clin Oncol 2002;20:1167-74.

10. Welsh J, Settle SH, Amini A, et al. Failure patterns in patients with esophageal cancer treated with definitive chemoradiation. Cancer 2012;118:2632-40.

11. Higuchi K, Komori S, Tanabe S, et al. Definitive chemoradiation therapy with docetaxel, cisplatin, and 5-fluorouracil (DCF-R) in advanced esophageal cancer: a phase 2 trial (KDOG 0501-P2). Int J Radiat Oncol Biol Phys 2014;89:872-9.

12. De B, Rhome R, Doucette J, et al. Dose escalation of definitive radiation is not associated with improved survival for cervical esophageal cancer: a National Cancer Data
Base (NCDB) analysis. Dis Esophagus 2017;30:1-10.

13. Nicolini G, Ghosh-Laskar S, Shrivastava SK, et al. Volumetric modulation arc radiotherapy with flattening filter-free beams compared with static gantry IMRT and 3D conformal radiotherapy for advanced esophageal cancer: a feasibility study. Int J Radiat Oncol Biol Phys 2012;84:553-60.

14. Welsh J, Gomez D, Palmer MB, et al. Intensity-modulated proton therapy further reduces normal tissue exposure during definitive therapy for locally advanced distal esophageal tumors: a dosimetric study. Int J Radiat Oncol Biol Phys 2011;81:1336-42.

15. Welsh J, Palmer MB, Ajani JA, et al. Esophageal cancer dose escalation using a simultaneous integrated boost technique. Int J Radiat Oncol Biol Phys 2012;82:468-74.

16. Fletcher GH. Clinical dose-response curves of human malignant epithelial tumours. Br J Radiol 1973;46:1-12.

17. Withers HR, Peters LJ, Taylor JM. Dose-response relationship for radiation therapy of subclinical disease. Int J Radiat Oncol Biol Phys 1995;31:353-9.

18. Smith SA, Fox MP. Radiation doses in esophageal cancer. J Thorac Dis 2019;11:5688-90.

19. Ren X, Wang L, Han C, et al. Retrospective analysis of safety profile of high-dose concurrent chemoradiotherapy for patients with oesophageal squamous cell carcinoma. Radiother Oncol 2018;129:293-9.

20. He L, Allen PK, Potter A, et al. Re-evaluating the optimal radiation dose for definitive chemoradiotherapy for esophageal squamous cell carcinoma. J Thorac Oncol 2014;9:1398-405.

21. Brower JV, Chen S, Bassetti MF, et al. Radiation Dose Escalation in Esophageal Cancer Revisited: A Contemporary Analysis of the National Cancer Data Base, 2004 to 2012. Int J Radiat Oncol Biol Phys 2016;96:985-93.

22. Song T, Liang X, Fang M, et al. High-dose versus conventional-dose irradiation in cisplatin-based definitive concurrent chemoradiotherapy for esophageal cancer: a systematic review and pooled analysis. Expert Rev Anticancer Ther 2015;15:1157-69.

23. Chang CL, Tsai HC, Lin WC, et al. Dose escalation intensity-modulated radiotherapy-based concurrent chemoradiotherapy is effective for advanced-stage thoracic esophageal squamous cell carcinoma. Radiother Oncol 2017;125:73-9.

24. Chen CY, Li CC, Chien CR. Does higher radiation dose lead to better outcome for non-operated localized esophageal squamous cell carcinoma patients who received 


\section{Page 12 of 12}

concurrent chemoradiotherapy? A population based propensity-score matched analysis. Radiother Oncol 2016;120:136-9.

25. Yu WW, Zhu ZF, Fu XL, et al. Simultaneous integrated boost intensity-modulated radiotherapy in esophageal carcinoma: early results of a phase II study. Strahlenther Onkol 2014;190:979-86.

26. Chen D, Menon H, Verma V, et al. Results of a Phase 1/2 Trial of Chemoradiotherapy With Simultaneous

Cite this article as: Zhao J, Lei T, Zhang T, Chen X, Dong J, Guan Y, Wang J, Wei H, Er P, Han D, Wei X, Guo Z, Du Q, Wang J, Liu N, Song Y, Yuan Z, Zhao L, Zhang W, Pang Q, Wang P. The efficacy and safety of simultaneous integrated dose reduction in clinical target volume with intensity-modulated radiotherapy for patients with locally advanced esophageal squamous cell carcinoma. Ann Transl Med 2020;8(18):1160. doi: $10.21037 /$ atm-20-4366

\section{Zhao et al. The efficacy and safety of SIR-IMRT in ESCC}

Integrated Boost of Radiotherapy Dose in Unresectable Locally Advanced Esophageal Cancer. JAMA Oncol 2019;5:1597-604.

27. Welsh JW, Seyedin SN, Allen PK, et al. Local Control and Toxicity of a Simultaneous Integrated Boost for Dose Escalation in Locally Advanced Esophageal Cancer: Interim Results from a Prospective Phase I/II Trial. J Thorac Oncol 2017;12:375-82. 